P10 EFFECT OF PIRFENIDONE ON GAS TRANSFER IN PATIENTS WITH IDIOPATHIC PULMONARY FIBROSIS

PM George, L Richardson, EA Renzoni, M Kokosi, TM Maher, AU Wells, F Chua. Royal Brompton and Harefield NHS Foundation Trust, London, UK

10.1136/thoraxjnl-2015-207770.147

Background Idiopathic pulmonary fibrosis (IPF) is a severe and progressive interstitial lung disease (ILD). Treatment with the anti-fibrotic agent Pirfenidone slows decline in forced vital capacity (FVC). Pulmonary vasculopathy is a relatively common and life-limiting complication of IPF and is frequently associated with a reduction in the diffusing capacity of the lung for carbon monoxide (DLco). However, it is not known what effect Pirfenidone may have on DLco.

Methods We performed a retrospective analysis of patients with diagnoses of IPF on long term Pirfenidone treatment. Lung function data were collected at treatment initiation and then at 12 months, (6-18 months). To assess for a treatment effect, similar data were also collected from an untreated control cohort of biopsy proven IPF patients from the pre-Pirfenidone era managed at the same centre. Data were analysed using Stata.

Results 138 patients were studied; $\mathrm{n}=66$ patients in the untreated control group and $n=72$ patients in the Pirfenidone treated group. The control group had a higher baseline predicted FVC $(74.8 \%$ v $67.5 \%)(\mathrm{p}<0.05)$ but baseline predicted DLco measurements were similar $(44 \%$ v $40 \%)(p=0.19) .12$ month relative FVC change was greater in the untreated group; 9.9\% $(273 \mathrm{~mL})( \pm 11.4 \%)$ versus $3.9 \%(123 \mathrm{~mL})( \pm 11.9 \%)(\mathrm{p}<$ 0.005). 12 month relative DLco decline was also greater in the untreated group; $16.4 \%( \pm 20.5 \%)$ versus $7.5 \%( \pm 17.6 \%)(\mathrm{p}<$ $0.01)$. In multivariate analyses, the effect of Pirfenidone treatment had a $6.7 \%$ impact on FVC change $(2.7-10.6)(\mathrm{p}<0.001)$ and a 9.0\% impact on DLco change $(2.5-15.5)(\mathrm{p}<0.01)$. Right ventricular systolic pressure correlated with baseline predicted DLco ( $\left.\mathrm{p}<0.005, \mathrm{r}^{2}=-0.14\right)$.

Discussion In this study we have demonstrated that over 12 months, Pirfenidone confers a reduction in gas transfer decline paralleling that seen for FVC. This treatment effect on DLco may be due to a combination of deceleration in ILD progression as well as attendant effects at the level of the pulmonary vasculature. This may have particularly relevance given the correlation between DLco and echocardiographic parameters of pulmonary vascular disease.

Conclusion Treatment of IPF with Pirfenidone markedly attenuates declines in gas transfer. This is of interest as it may provide insights into mechanisms underpinning disease stabilisation.

\section{P11 PIRFENIDONE TREATMENT IS ONLY AVAILABLE IN THE UK FOR A MINORITY OF PATIENTS WITH USUAL INTERSTITIAL PNEUMONITIS}

G Burge, D Petkova, S Ghani, J Reynolds, M Djearman, E Hoey, S Hussain, PS Burge. Heart of England NHS Foundation Trust, Birmingham, UK

\subsection{6/thoraxjnl-2015-207770.148}

Introduction Usual Interstitial Pneumonitis (UIP) may be caused by asbestos exposure (asbestosis), collagen vascular diseases (CVD) or agents causing hypersensitivity pneumonitis (HP), or may be idiopathic (IPF). Referrals to our ILD MDT have increased since the limited availability of pirfenidone which is only prescribable for IPF with a FVC $50-80 \%$ predicted. We have reviewed presentation in 2014 to identify patients suitable for Pirfenidone prescription.

Methods Our hospital provides a regional service for Pirfenidone prescription. All patients with a MDT diagnosis of definite UIP were included. A standard proforma requested information on exposures, CVD and antibodies relevant to CVD and HP. If there were no relevant exposures and CVD was excluded, a diagnosis of IPF was made. Asbestosis, HP and CVD associated UIP were diagnosed when the relevant information supported this, unspecified UIP was diagnosed in the absence of specific information.

Results 202/546 referrals in 2014 were judged to have definite UIP after consideration by a fully constituted ILD MDT including histopathologists, radiologists, clinicians and CNS'. After exclusion of 22 with asbestosis, 11 with CVD associated UIP, 17 with CPFE and 17 with HP associated UIP, 51 with IPF and 84 with unspecified UIP remained for consideration of Pirfenidone treatment. Only 64/135 suitable patients had a FVC 50-80\% predicted (Figure 1).

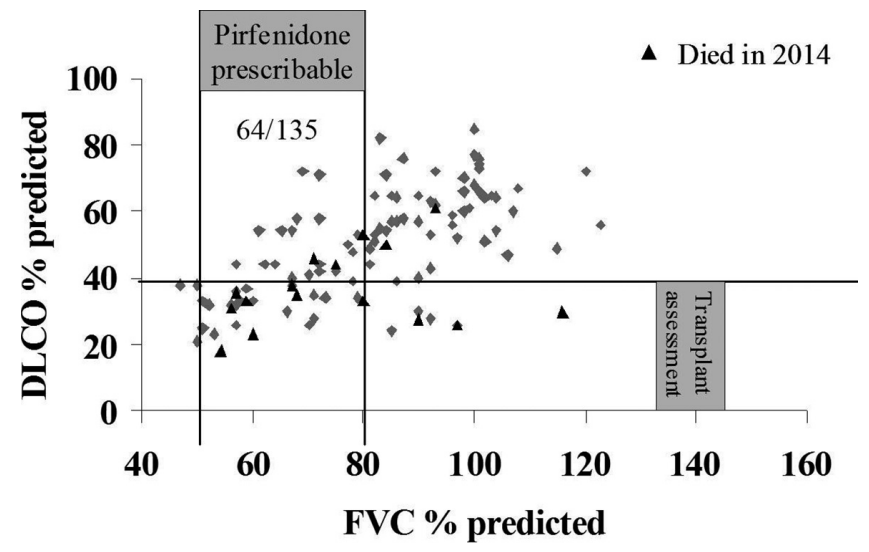

\section{Abstract P11 Figure 1}

Conclusions The decisions of an ILD MDT are limited by the completeness of investigation. We found causes for 50/200 patients with UIP. Pirfenidone was not prescribable for $53 \%$ of otherwise suitable patients. If the FVC limit was raised to $90 \%$ $32 \%$ would still be excluded, including 2 who died of their disease within 12 months. The FVC is often preserved even in terminal IPF.

\section{P12 PIRFENIDONE POST-AUTHORISATION SAFETY REGISTRY (PASSPORT) - UPDATE AND CONCOMITANT USE OF N- ACETYLCYSTEINE AND/OR CORTICOSTEROIDS}

${ }^{1} \mathrm{~T}$ Maher, ${ }^{2} \mathrm{~V}$ Cottin, ${ }^{3} \mathrm{~A}$ Azuma, ${ }^{4} \mathrm{~L}$ Groves, ${ }^{4} \mathrm{P}$ Hormel, ${ }^{5} \mathrm{M}$ Sköld, ${ }^{6} \mathrm{~S}$ Tomassetti, ${ }^{7} \mathrm{D}$ Koschel. ${ }^{1}$ Royal Brompton Hospital, London, UK; ${ }^{2}$ National Reference Center for Rare Pulmonary Disease, Louis Pradel Hospital, Lyon, France; ${ }^{3}$ Nippon Medical School, Tokyo, Japan; ${ }^{4}$ Genentech, South San Francisco, CA, USA; ${ }^{5}$ Karolinska Institute, Stockholm, Sweden; ${ }^{6} G B$ Morgagni Hospital, Forli, FC, Italy; ${ }^{7}$ Fachkrankenhaus Coswig, Coswig, Germany

10.1136/thoraxjnl-2015-207770.149

Background PASSPORT is a post-authorisation safety registry for pirfenidone to collect real-world data in EU patients with idiopathic pulmonary fibrosis (IPF). This analysis assessed the safety of pirfenidone as monotherapy and in combination with $\mathrm{N}$-acetylcysteine (NAC) and/or corticosteroids (CS). 